\title{
Estudio experimental de la respuesta geomecánica de relaves en pasta cementados utilizados para el relleno de caserones
}

\section{Experimental study of the geomechanical response of cemented paste backfills used in} underground mining

Fecha de entrega: 12 de enero 2015 Fecha de aceptación: 4 de mayo 2015

\section{Gonzalo Suazo, Andy Fourie y Alsidqi Hasan}

School of Civil, Environmental and Mining Engineering, The University of Western Australia, 35 Stirling Highway, Crawley WA 6009, Australia, gonzalosuazo@gmail.com, andy.fourie@uwa.edu.au, alsidqi.hasan@uwa.edu.au

La tecnología de relleno de caserones con relaves en pasta cementados se ha popularizado en la industria minera subterránea a nivel mundial. Esta tecnología brinda una serie de ventajas desde el punto productivo y medio ambiental. Sin embargo, una serie de fallas en las barreras utilizadas para contener el relleno se han registrado en las últimas décadas. En este artículo se presentan los resultados de la instrumentación geotécnica de un caserón real, la que permitió cuantificar las presiones horizontales totales y presiones de poros tanto al interior del relleno como en la barreras. Se encontró que altas presiones de poros y presiones horizontales se desarrollan en las primeras etapas de llenado. Sin embargo, periodos de descanso entre etapas favorecen la consolidación del relleno y la generación de efecto de arco entre el relleno y la roca, lo que reduce la tasa de incremento de las presiones sobre la barrera en etapas posteriores de llenado. Adicionalmente, se observó que cargas dinámicas pueden aumentar considerablemente la presión de poros al interior del relleno. En este contexto, al término del artículo se presentan resultados experimentales respecto al potencial de licuefacción de rellenos cementados.

Palabras clave: relleno de caserones, relaves cementados, presión de poros, presión horizontal total, licuefacción
Cemented paste backfill CPB has been widely used in underground mining operations in the recent past for filling the voids (stopes) left after blasting and mucking of ore. It provides a series of advantageous environmental, safety and economic characteristics. However, several barricades failures have been reported in the recent past. This paper presents the results of in situ monitoring carried out to quantify pore pressure and total horizontal stresses exerted on barricades. It was found that high horizontal stresses and pore pressures develop during the early filling stages. Resting periods were shown to effectively improve the consolidation of the fill and the arching effect on the interface fill-rock. These effects seem to reduce the rate of increment of horizontal stresses acting on barricades at later filling stages. In addition, it was observed that dynamic loads can significantly increase pore pressure within the fill. In this context, experimental results about the liquefaction potential of CPB are provided.

Keywords: Cemented Paste Backfill, barricades, pore pressure, total horizontal stresses, liquefaction

\section{Introducción}

La tecnología de relleno minero con relaves en pasta cementados CPB (Cemented Paste Backfill) ha sido ampliamente utilizada en las últimas décadas con el objetivo de rellenar las grandes perforaciones dejadas luego de las operaciones de tronadura ejecutadas para la extracción subterránea de minerales. El uso de esta técnica tiene una serie de ventajas respecto a otros métodos de extracción, por ejemplo, provee una mayor estabilidad local y global a la mina, permite la extracción de mineral desde los pilares ubicados entre caserones y brinda la posibilidad a ingenieros geotécnicos de depositar una gran cantidad de relaves bajo tierra, lo que disminuye los riesgos asociados a la disposición en superficie y el impacto ambiental de la operación minera (Helinski, 2008).

La técnica consiste en rellenar los caserones con una mezcla de relaves integrales, agua y cemento con una concentración de sólidos (peso de sólidos sobre peso total) que típicamente 
varía entre 75 y $85 \%$ y un contenido de cemento de 3 a $7 \%$ respecto al peso total seco (Benzaazoua et al., 2004). Otra práctica común es agregar un porcentaje de arena a la mezcla con el objetivo de incrementar la permeabilidad y mejorar la consolidación del relleno (Potvin et al., 2005). Durante las primeras horas de depositación, la mezcla se comporta como un fluido no Newtoniano, sin embargo, en la medida que el cemento se hidrata, el relleno se comporta como un sólido. Inicialmente, una barrera es construida en la base del caserón para contener la mezcla (Figura 1a). Estas barreras deben resistir la presión total horizontal de la mezcla $\sigma_{\mathrm{h}}$, así como también las cargas dinámicas generadas por eventos sísmicos (estallidos de roca, sismos) y operaciones de tronadura. Este tipo de cargas generan un incremento en la presión de poros $u$ lo que reduce la presión efectiva vertical $\sigma{ }_{\mathrm{v}}$. En términos de la teoría de Rankine, la cual sin embargo es una teoría simplificada para este tipo de escenarios (dada la anisotropía del relleno, efecto arco y desplazamiento lateral de la roca confinante, entre otras); la presión horizontal en una barrera está dada por:

$$
\sigma_{h}=\sigma_{v}^{\prime} K_{0}+u
$$

donde $K_{0}$ es el coeficiente de empuje en reposo. Una serie de fallas de estas estructuras, con el consecuente flujo de la mezcla al interior de las áreas de producción, se han registrado en las últimas décadas (Revell y Sainsbury, 2007). Estas fallas no sólo tienen un costo económico importante (dados los retrasos en las operaciones de extracción), sino que también generan un alto riesgo en la seguridad del personal. Para reducir los efectos de cargas estáticas y dinámicas en la estructura de contención, una de las estrategias generalmente utilizada consiste en rellenar el caserón en etapas. Un primer "tapón" de mezcla es depositado hasta un metro sobre la barrera, para continuar con un periodo de reposo (v.g. $24 \mathrm{hr}$ ), durante el cual la mezcla consolida y el cemento se hidrata. Finalmente, el caserón se termina de rellenar hasta el techo, o bien se rellena hasta la base de la siguiente barrera donde se permite otro periodo de reposo.

Una serie de ecuaciones simplificadas se han propuesto para estimar las presiones horizontales totales en las paredes de los caserones y en particular en las barreras, ya sea en condición drenada o no drenada (Aubertin et al., 2004). Sin embargo, estas ecuaciones no logran incorporar del todo una serie de fenómenos que ocurren al interior del caserón y que podrían, eventualmente, afectar la estabilidad de las barreras, v.g. efectos de cargas dinámicas, fricción en las paredes del caserón, inclinación del relleno y efectos químicos de la hidratación el cemento. Más aún, son escasos los estudios experimentales in situ y de laboratorio para validar dichas ecuaciones.

En este contexto, en el proyecto Mine Backfill liderado por la Universidad de Western Australia, se ha instrumentado una serie de caserones localizados en diferentes minas en Australia. El objetivo de este proyecto es evaluar las presiones totales y presiones de poro producidas en condiciones normales de operación, tanto en las estructuras de contención como en distintas locaciones al interior del relleno. Los resultados de la instrumentación realizada en una mina de oro de pequeñas dimensiones son presentados en este artículo. Adicionalmente, se presentan resultados de la investigación llevada a cabo por los autores para la evaluación del potencial de licuefacción de relaves en pasta cementados.

\section{Monitoreo de caserones Instrumentación}

La instrumentación se ejecutó en un caserón que presentaba una única barrera de contención en su altura (Figura 1a) y de dimensiones en planta de $18 \times 3.5 \mathrm{~m}$ y altura aproximada de $17 \mathrm{~m}$. El caserón estaba inclinado respecto a la horizontal en $65^{\circ}$ (Figura 1b) y presentaba una gran irregularidad e inestabilidad en sus paredes, lo que hizo frecuente los desprendimientos de roca durante la ejecución de la etapa experimental. La barrera no permitía el flujo de agua desde el relleno (impermeable) y la roca del caserón presentaba una muy pequeña permeabilidad, por lo que el agua del relleno drenaba sola en la dirección vertical.

Una celda de carga para la medición de presiones totales, del tipo Geokon NATM con una capacidad máxima de 700 $\mathrm{kPa}$, fue instalada entre la pasta y la barrera en lo que se denomina como locación L2 en la Figura 1a. La barrera fue construida mediante el método del arco, que consiste básicamente en aplicar shotcrete a un marco metálico con malla de acero que está arqueado y apernado a las paredes del drive de acceso. De este modo, para asegurar una buena superficie de contacto para la celda de carga, una placa metálica de 40x30 cm fue montada sobre la barrera. 
Dos piezómetros para la medición de presión de poros del tipo Geokon modelo 1200 con capacidad máxima de 700 $\mathrm{kPa}$ fueron localizados al interior del caserón L1 y en la interfaz relleno-barrera L2. Ambos tipos de instrumentos registran presiones o esfuerzos mediante sensores de cuerda vibrante y termistor. Las mediciones fueron recolectadas por un data logger localizado en el drive superior. Ningún instrumento fue instalado al interior del relleno (sección central) producto de las condiciones de operación y seguridad de la mina.

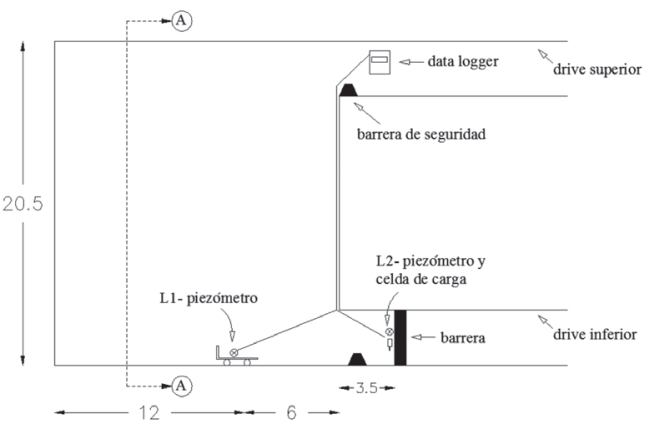

(a)

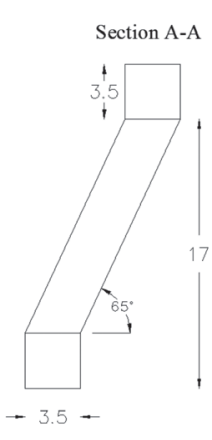

(b)
Figura 1: Ubicación de sensores en caserón, a) vista en elevación y b) corte A-A. Dimensiones en metros.

\section{Materiales}

El relleno utilizado consistía en una mezcla de relaves, arena, cemento y agua obtenida del proceso de extracción de mineral. El agua presentaba una gran concentración de sales con un total de sólidos disueltos TDS de 103.000 $\mathrm{ppm}$. Una alta concentración de sales podría eventualmente afectar la cementación del relleno y su estabilidad en el largo plazo (Hasan et al., 2014). Como aditivo se utilizó cemento Portland ordinario. La mezcla se preparó con un $75 \%$ de sólidos, un $5 \%$ de cemento y un $30 \%$ de arena (del peso total). Como resultado, la mezcla final poseía una densidad aproximada de $1.93 \mathrm{ton} / \mathrm{m}^{3}$ y una resistencia a compresión no confinada de $210 \mathrm{kPa}$ a los 4 días de curado. La distribución granulométrica para los relaves, arena y mezcla se presenta en la Figura 2. Los relaves presentaban un peso específico $G_{\mathrm{s}}$ de 2.75 , con un porcentaje de finos superior al $39 \%$ y un coeficiente de uniformidad $C_{\mathrm{u}}$ de 50.6 .

\section{Secuencia de llenado}

El caserón se rellenó a una tasa de $110 \mathrm{~m}^{3} / \mathrm{hr}$, lo que se traduce en un ritmo de llenado de $1.7 \mathrm{~m} / \mathrm{hr}$. La mezcla

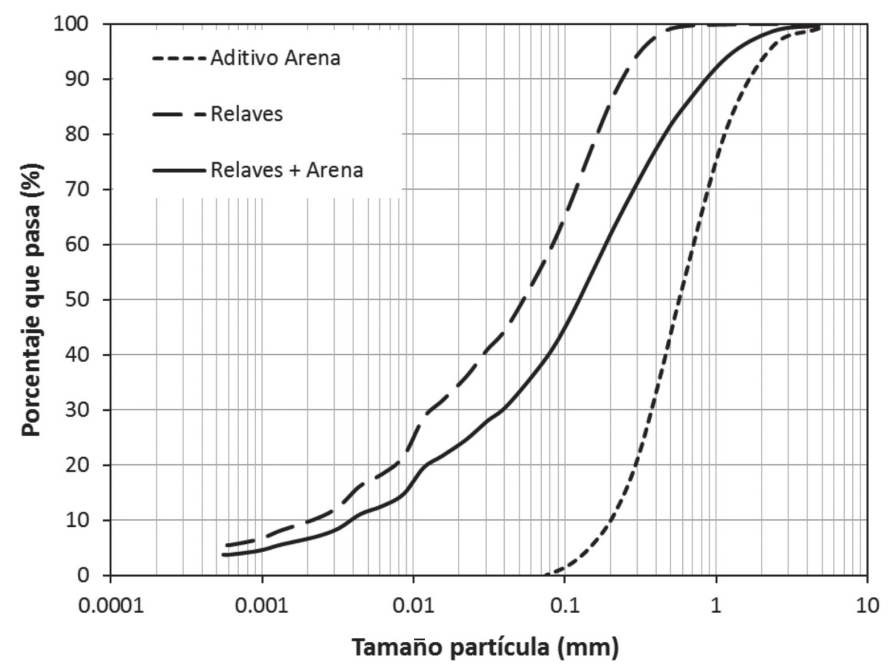

Figura 2: Distribución granulométrica de materiales utilizados en relleno de caserón

fue transportada desde la planta de mezclado, ubicada en superficie, hasta los caserones mediante bombas de alta presión y tuberías de HDPE. Debido a las pequeñas dimensiones de los caserones de esta mina, solo dos etapas de llenado y una de reposo son necesarias para completar el proceso de relleno. En una primera etapa, se llena hasta la parte superior de la barrera hasta una elevación aproximada de $5.9 \mathrm{~m}$ (Figura 3), luego de lo cual se permite un periodo de reposo de $24 \mathrm{hr}$. Finalmente, se termina de rellenar el caserón hasta la base de la siguiente barrera (elevación $17 \mathrm{~m}$ ). Dado que el relleno se utiliza como plataforma de trabajo para la extracción del caserón contiguo, no se rellena hasta el techo del caserón.

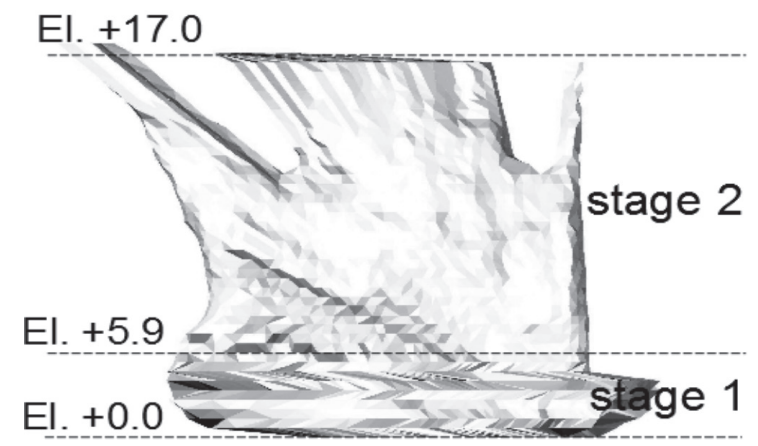

Figura 3: Elevaciones aproximadas en etapas de llenado

\section{Resultados y discusión}

En la Figura 4a se muestran las mediciones registradas por los tres sensores. También en estas figuras se encuentra la curva de esfuerzo geostático vertical total, dada por el producto del peso unitario total $\gamma$ del relleno y la altura relativa $h_{\mathrm{r}}$ entre el relleno y el nivel de instalación de los 
instrumentos. La altura del relleno se calculó a partir de la geometría del caserón, obtenida de mediciones CMS (Sistema de Monitoreo de Cavidades) (Figura 3), y la tasa de llenado impuesta por la planta de mezclado.

(a)

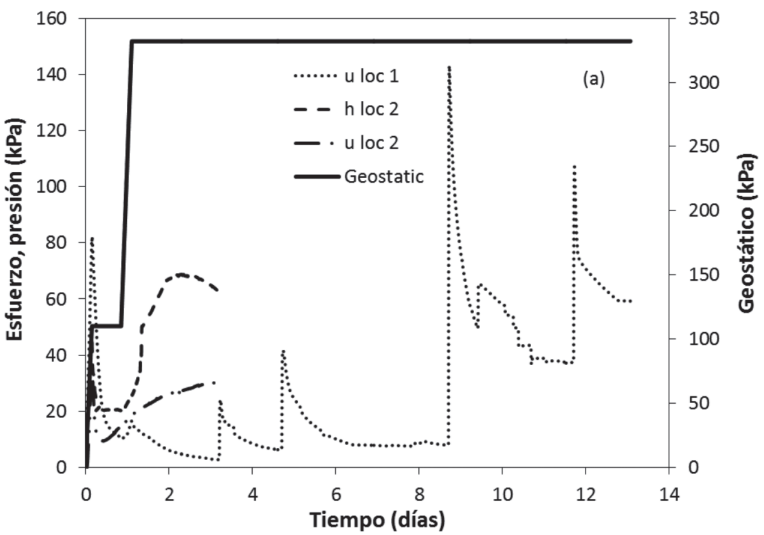

(b)

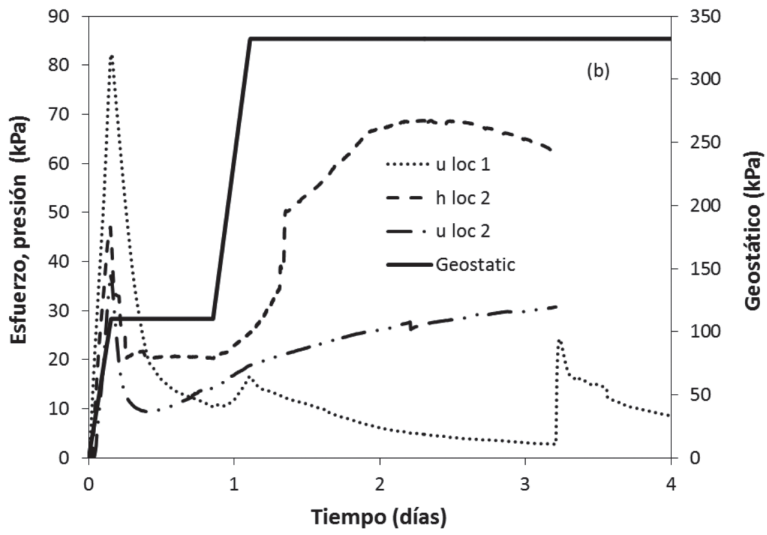

Figura 4: Presiones horizontales totales $\sigma_{h}$ y presiones de poros $u$ registradas en caserón instrumentado. Mediciones registradas durante los primeros (a) 14 y (b) 3.7 días de depositación.

Las mediciones fueron registradas por un periodo de 13 días, periodo durante el cual los sistemas de monitoreo sísmico subterráneo registraron una serie de eventos sísmicos de pequeña intensidad. Asimismo, las operaciones de tronadura de caserones contiguos fueron reiniciadas luego del tercer día de depositación. Los efectos dinámicos de estas actividades se traducen en un aumento considerable de la presión de poros como se observa en las mediciones registradas por el piezómetro en L1. En esta figura se observan al menos 4 máximos de incremento de presión de poros, los que son coincidentes con los tiempos en los cuales las tronaduras fueron programadas. Los otros pequeños aumentos de presión de poros son atribuidos a las actividades sísmicas de campo cercano registradas durante el periodo de medición. Es interesante observar que un gran incremento de presión de agua se produce a los 9 días, cercano a los $140 \mathrm{kPa}$, a pesar del avanzado grado de cementación del relleno.

El piezómetro en L1 registró continuamente durante los 13 días, sin embargo, el piezómetro y celda de carga en L2 mostraron mediciones erróneas luego de 3.7 días. Este problema de registro se atribuyó a un daño permanente de los instrumentos debido a las actividades de tronadura. La programación de extracción y tronadura contemplaba la extracción del caserón en el nivel inferior, muy cercano a la barrera del caserón en estudio, lo que podría haber dañado parcialmente el relleno y por ende los instrumentos en L2.

En la Figura 4b el rango de medición se ha acotado a 3.7 días para una mejor comparación de resultados. En general, se observa que hay un aumento consistente en las mediciones de los distintos instrumentos en los periodos de llenado producto del aumento en la presión vertical total. Sin embargo, durante los periodos de descanso tanto la presión de poros como la presión horizontal disminuyen producto de la consolidación del relleno y de la fricción generada entre las paredes del caserón y el relleno (Fahey et al., 2009). Luego del periodo de reposo, la respuesta de la presión de poros y presión horizontal es menos pronunciada, lo que se atribuye a una mayor rigidez del relleno dada la consolidación e hidratación del cemento. Una vez concluida la última etapa de llenado, las lecturas en los piezómetros comienzan a disminuir, lo que se atribuyó a la consolidación del relleno y al efecto conocido como self dessication, correspondiente a una reducción de la presión de poros producto del consumo de agua para la formación de los subproductos del cemento (Grabinsky y Simms, 2005). Como evidencia del proceso exotérmico de hidratación del cemento, sensores de temperatura instalados al borde del caserón registraron un cambio de temperatura de $23^{\circ} \mathrm{C}$ al inicio de la depositación a $33^{\circ} \mathrm{C}$ al término del proceso de monitoreo.

Durante las primeras horas de depositación, en el cual el relleno recién comienza a consolidar, la presión de poros y presión horizontal en L2 son similares. En este sentido, una mejor comparación de mediciones puede ser obtenida utilizando los índices de esfuerzo total $\left(T S R=\sigma_{h} / \gamma h_{r}\right)$ e índice de presión de poros $\left(P P R=u / \gamma h_{r}\right)$. Estos índices son graficados versus la altura total del relleno $h_{r}$ en la Figura 5a para las distintas etapas de llenado. 
(a)

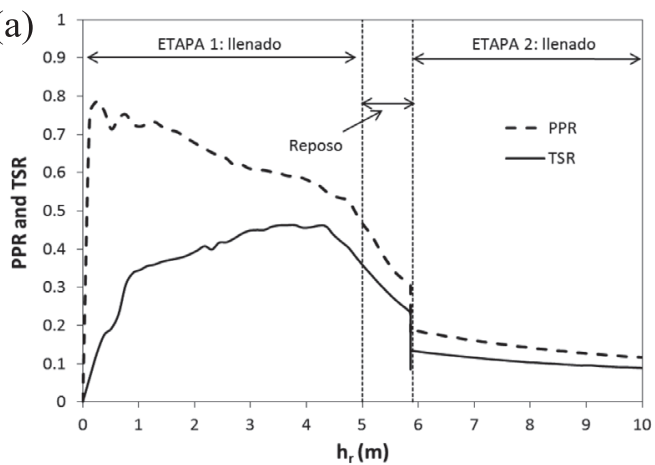

(b)

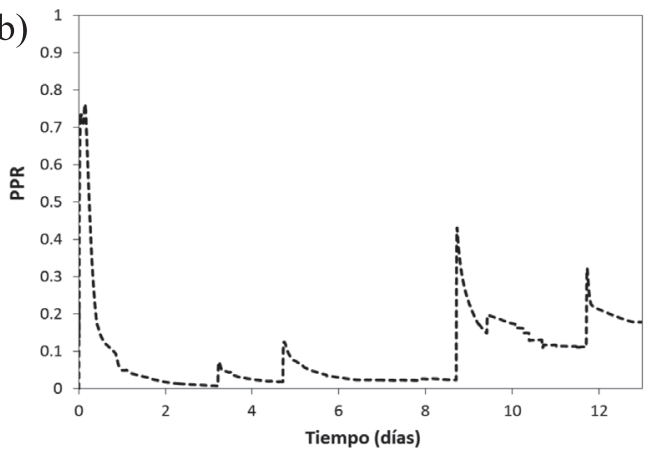

Figura 5: a) Índices de presión de poros y esfuerzo total en barrera L2 y b) índice de presión de poros para el periodo de mediciones en L1

El máximo valor del índice de presión de poros, alrededor de 0.8 , ocurre en las primera horas de depositación, cuando el relleno alcanza $0.4 \mathrm{~m}$, y tiende a disminuir ligeramente al término de la primera etapa de 1lenado. Durante el periodo de descanso la presión de poros disminuye considerablemente y $P P R$ llega a un valor de 0.2. En la segunda etapa de llenado, $P P R$ se mantiene prácticamente constante llegando a un valor mínimo de 0.16. Valores de $P P R$ cercanos a 1 indicarían que gran parte de la presión vertical total se transfiere en forma de presión de poros a la barrera. El cambio drástico y permanente en $P P R$ luego del término de la primera etapa de llenado, valida la eficacia del método de depositación en etapas y en particular el uso de un "tapón" inicial sobre la barrera. De manera similar, el índice de esfuerzo total TSR, aumenta en la medida que el relleno crece en altura, alcanzando un valor máximo de 0.4 al término de la primera etapa de llenado. Como sucede con la presión de poros, existe una rápida disminución del $T S R$ en la etapa de reposo del relleno. Luego de esta etapa, el TSR alcanza un valor mínimo de 0.16 , lo que se traduce en un aumento menos pronunciado en el esfuerzo horizontal total en la barrera en la medida que el relleno crece en altura (Figura 5a).
Para ilustrar los efectos de cargas dinámicas sobre la presión de poros el valor de $P P R$ versus tiempo de curado ha sido graficado en la Figura $5 b$. De esta curva se puede reconocer que existe un importante efecto de cargas dinámicas sobre la presión total ejercida por el relleno sobre la barrera. Se observan valores de $P P R$ por sobre 0.4 a los 9 días, tiempo en el cual la presión de poros debiese ser similar a la presión efectiva de confinamiento vertical (falla por licuefacción), asumiendo que el relleno ha consolidado completamente. Para este caserón, las ondas de compresión debido a procesos de tronadura resultan más importantes que las ondas de corte producto de fenómenos sísmicos. Sin embargo, dependiendo de las condiciones geológicas, los esfuerzos de corte podrían también tener un efecto importante sobre el aumento de la presión de poros del relleno. En este contexto, en los siguientes acápites se proporcionan resultados experimentales sobre la susceptibilidad a la licuefacción sísmicamente inducida de relaves en pasta cementados.

\section{Comportamiento cíclico de relaves integra- les cementados}

Una serie de ensayos de corte simple directo cíclico (Direct Simple Shear Test) fueron llevados a cabo en relaves integrales de plata-plomo-zinc cementados. El equipo utilizado fue manufacturado por GEOCOMP (Figura 6) y es del tipo desarrollado en NGI (Norwegian Goetechnical Institute) a mediados de los años 60. El ensayo genera un estado homogéneo de esfuerzos de corte en la muestra bajo condiciones normales de consolidación y permite reproducir la rotación de esfuerzos principales durante la carga cíclica. En general, es un ensayo más realista que técnicas tradicionales de medición de resistencia cíclica, como el ensayo triaxial. Este equipo ha sido utilizado en otros estudios para la evaluación de la resistencia cíclica de relaves finos (Wijewickreme et al., 2005; Al-Tarhouni et al., 2011). Las muestras fueron preparadas mediante el método de depositación de slurry, propuesto por Kuerbis y Vaid (1998). Se produjeron muestras con una altura de 27 mm y un diámetro de $72 \mathrm{~mm}$, aproximadamente.

Los relaves utilizados correspondían a limos de baja plasticidad (ML) con un contenido de finos superior al $50 \%$. Las muestras fueron preparadas con contenidos de cemento de un $5 \%$, contenido de sólidos del $78 \%$ y curadas 
por un periodo máximo de $24 \mathrm{hr}$. En la Figura 7 se presentan los resultados de un ensayo cíclico a niveles de presión efectiva de $100 \mathrm{kPa}$ para una muestra curada por $12 \mathrm{hr}$. En general, las muestras presentaron falla por movilidad cíclica y ninguna de las muestras ensayadas presentó falla de flujo. La resistencia cíclica aumentó considerablemente en la medida que la hidratación del cemento avanzaba, debido a la cementación de los poros del relleno.

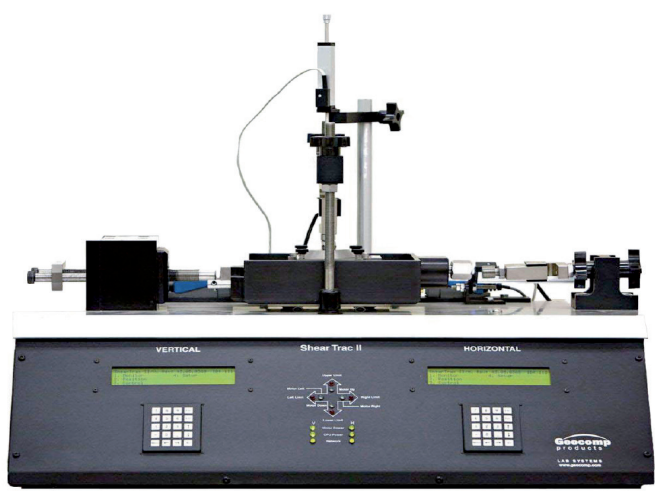

Figura 6: Equipo GEOCOMP utilizado para ensayos de resistencia cíclica de relaves cementados
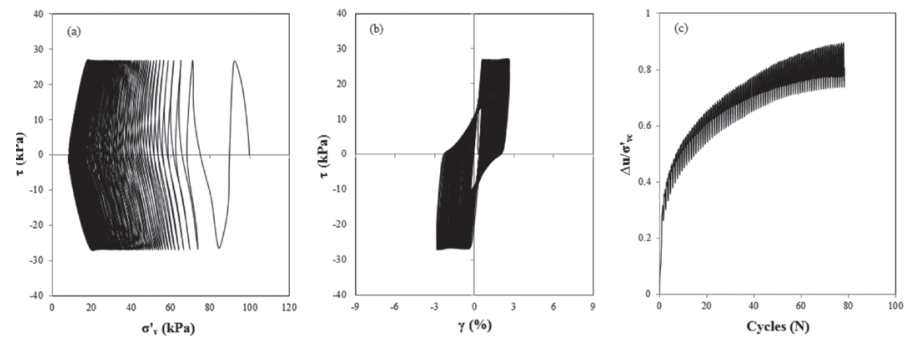

Figura 7: Respuesta cíclica de relaves integrales cementados en equipo de corte simple directo GEOCOMP $\left(\sigma^{\prime}{ }_{\mathrm{vc}}=100 \mathrm{kPa}, \mathrm{CSR}\right.$ $=0.27,5 \%$ cemento, $12 \mathrm{hr}$ curado). (a) Presión de confinamiento $\sigma^{\prime}{ }_{\text {vc }}$ versus esfuerzo de corte $\tau$, (b) deformación de corte $\gamma$ versus esfuerzo de corte $\tau$ y (c) número de ciclos $\mathrm{N}$ versus razón de presión poros $\Delta \mathrm{u} / \sigma_{v c}^{\prime}$.

La Figura 8a presenta los resultados de 18 de estos ensayos en términos de la razón de tensiones cíclica solicitante CSR versus el número de ciclos $\mathrm{N}$ para cumplir con el criterio de falla de licuefacción. CSR se define como la razón entre el corte cíclico $\tau_{\text {cy }}$ y la presión efectiva de confinamiento $\sigma^{\prime}{ }_{v c}$. Se utilizó como criterio de falla un 3.75\% de deformación en amplitud simple. A este nivel de deformación, se alcanzó en promedio un máximo incremento de presión de poros $\Delta \mathrm{u}$ de aproximadamente $90 \%$ de la presión efectiva de confinamiento (i.e. razón de presión de poros).
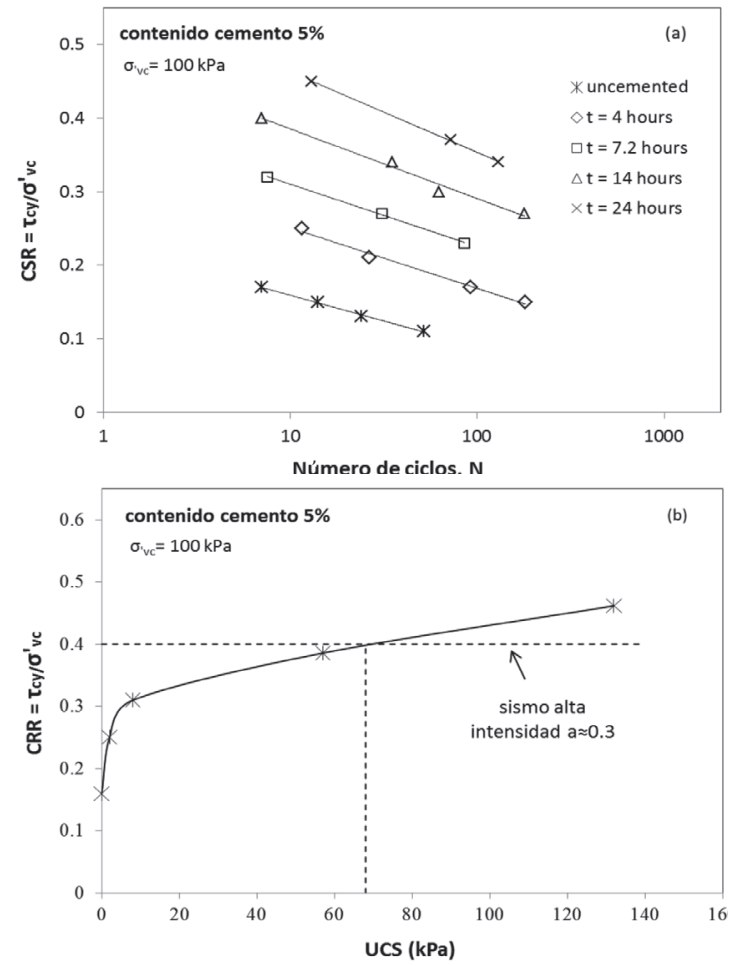

Figura 8: a) CSR versus número de ciclos para alcanzar un $3.75 \%$ de deformación en amplitud simple y b) CRR a 10 ciclos versus resistencia a compresión no confinada.

La Figura 8a muestra que a temprana edad, el relleno es más susceptible a experimentar falla por licuefacción. Asimismo, muestras curadas por periodos más largos requieren de una cantidad mayor de ciclos para aumentar la presión de poros a un mismo CSR. En la Figura 8b, la razón de resistencia cíclica a 10 ciclos a distintas edades se comparó con la resistencia a compresión no confinada UCS del relleno a dicha edad. En el mismo gráfico, se definió una línea representando un sismo de alta intensidad capaz de generar una CSR de 0.4. Se observa que luego de que el relleno alcanza una resistencia no confinada de $65 \mathrm{kPa}$ el riesgo de falla por licuefacción desaparece. Esta resistencia no confinada se alcanza en un periodo de $15 \mathrm{hr}$. En la práctica, ingenieros geotécnicos definen periodos de reposo para el relleno de entre 12 y $48 \mathrm{hr}$ (dependiendo del contenido de cemento y tamaño del caserón), con el objetivo de alcanzar UCS en el orden de los $100 \mathrm{kPa}$. Luego de los resultados de esta investigación, esta práctica parece ser apropiada solo bajos eventos de muy alta intensidad. Sin embargo, considerando los eventos sísmicos típicamente observados en minería subterránea, esta práctica parece ser altamente conservadora. 


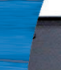

\section{Conclusiones}

En esta investigación las presiones generadas en la barrera utilizada para contener el relleno cementado al interior del caserón fueron analizadas mediante la instrumentación de un caserón real. Se encontró que tanto la presión total horizontal, como la presión de poros aumentan al incrementarse la altura del relleno en la primera etapa de llenado. Sin embargo, los periodos de reposo entre etapas pueden considerablemente disminuir esta tendencia en etapas de llenado posteriores.

Por otro lado, se encontró que cargas dinámicas pueden tener un importante efecto sobre las presiones máximas sobre la barrera. Un considerable aumento en la presión de poros debido a esfuerzos dinámicos se observa inclusive a edades de curado por sobre los 9 días. Estos aumentos se atribuyen a cargas generadas por sismos y por operaciones de tronadura.

Al estudiar el potencial de licuefacción de rellenos conformados por relaves integrales finos, se encontró que el relleno en general experimenta falla por movilidad cíclica con incrementos de presión de poros que pueden alcanzar el 90\% de la presión efectiva de confinamiento. En la medida que el cemento dentro del relleno se hidrata, la resistencia cíclica aumenta de manera considerable. Asimismo, se encontró que por sobre valores de resistencia no confinada de $65 \mathrm{kPa}$ el riesgo de experimentar una falla por licuefacción es muy bajo.

\section{Agradecimientos}

Los autores agradecen el financiamiento otorgado por ARC Linkage Project (LP100200173) a través de The University of Western Australia. El primer autor también agradece el financiamiento otorgado por la Australian International Postgraduate Research Scholarships (IPRS) y la Comisión Nacional de Investigación Científica y Tecnológica de Chile (CONICYT PAI/INDUSTRIA 79090016) para proseguir estudios de doctorado en el extranjero y agradece en forma especial a Geotecnia Ambiental Ltda.

\section{Referencias}

Al-Tarhouni, M., Simms, P. and Sivathayalan, S. (2011). Cyclic behaviour of reconstituted and dessicated-rewet thickened gold tailings in simple shear. Canadian Geotechnical Journal 48(7), 1044-1060

Aubertin, M., Li, L., Arnoldi, S., Belem, T., Bussière, B., Benzaazoua, M. and Simon, R. (2004). Interaction between backfill and rock mass in narrow stopes. 39th U.S. Rock Mechanics Symposium: 12th Panamerican Conference on Soil Mechanics and Geotechnical Engineering, Boston, Massachusetts, USA

Benzaazoua, M., Fall, M. and Belem, T. (2004). A contribution to understanding the hardening process. Minerals Engineering 17 (2), 141-152

Fahey, M., Helinski, M. and Fourie, A. (2009). Some aspects of the mechanics of arching in backfilled stopes. Canadian Geotechnical Journal 46(11), 1322-1336

Grabinsky, M.W. and Simms, P. (2005). Matric suction generated by self-desiccation in cemented paste backfill. Saskatoon, Sask. In: Proceedings of the $58^{\text {th }}$ Canadian Geotechnical Conference, 18-21.

Hasan, A., Suazo, G., Doherty, J. and Fourie, A. (2014). Case study: In-stope measurements at two Western Australian mine sites. Toronto, Canada, $17^{\text {th }}$ International Seminar on Paste and Thickened Tailings: Paste 2014

Helinski, M. (2008). Mechanics of mine backfill. PhD thesis, The University of Western Australia, Perth, Australia

Kuerbis, R. and Vaid, Y.P. (1998). Sand sample preparation - The slurry deposition method. Soils and Foundations 28(4), 107-118

Potvin, Y., Thomas, E. and Fourie, A. (2005). Handbook on Mine Fill. Australian Centre of Geomechanics, Perth, Australia.

Revell, M.B. and Sainsbury, D.P. (2007). Paste Bulkhead Failures. Melbourne, Victoria, Australia, Minefill 2007

Wijewickreme, D., Sanin, M.V. and Greenaway, R. (2005). Cyclic shear response of fine-grained mine tailings. Canadian Geotechnical Journal 42(5), 1408-1421 